\title{
Criminologie
}

\section{Du côté des victimes, une autre perspective sur le vol à main armée}

\section{Micheline Baril et Anne Morrissette}

Volume 18, numéro 2, 1985

Le vol à main armée à Montréal

URI : https://id.erudit.org/iderudit/017219ar

DOI : https://doi.org/10.7202/017219ar

Aller au sommaire du numéro

\section{Éditeur(s)}

Les Presses de l'Université de Montréal

ISSN

0316-0041 (imprimé)

1492-1367 (numérique)

Découvrir la revue

Citer cet article

Baril, M. \& Morrissette, A. (1985). Du côté des victimes, une autre perspective sur le vol à main armée. Criminologie, 18(2), 117-133.

https://doi.org/10.7202/017219ar
Résumé de l'article

Armed robbery has long been regarded as a crime against property. But from the victim's point of view, it is a violent crime which endangers their lives and constitutes a traumatic experience.

Nine years of research are briefly summarized with special attention to a recent survey of victims of commercial robbery in Montreal in which 440 persons were interviewed. It is difficult to describe a victim unless researchers agree on some basic definition of who should be defined as a victim. This is the first subject of discussion. After a short description of the way victimizations occur, the consequences of the robbery are discussed, and the responses of the mental health and justice systems are presented.

Most victims do not resist and those who do so seem to be reacting to past victimizations or to an excess of violence on the part of the robber. Nearly $90 \%$ of victims suffer some kind of emotional trauma and far from being helped in this regard, this trauma is often aggravated by the criminal justice system's response. It seems to affect the victim much more than the financial, physical and social consequences of the crime, which had little effect on their attitudes and needs.

The main problem with armed robbery is that it creates and perpetuates a climate of suspicion, fear and anger very damaging to social relationships. These negative effects can be reduced, however, and the study points out some of the means by which this can be accomplished.
Ce document est protégé par la loi sur le droit d'auteur. L’utilisation des services d’Érudit (y compris la reproduction) est assujettie à sa politique d'utilisation que vous pouvez consulter en ligne.

https://apropos.erudit.org/fr/usagers/politique-dutilisation/ 
DU CÔTÉ DES VICTIMES. UNE AUTRE PERSPECTIVE SUR LE VOL À MAIN ARMÉE Micheline Baril* et Anne Morissette**

Armed robbery has long been regarded as a crime against property. But from the victim's point of view, it is a violent crime which endangers their lives and constitutes a traumatic experience.

Nine years of research are briefly summarized with special attention to a recent survey of victims of commercial robbery in Montreal in which 440 persons were interviewed. It is difficult to describe a victim unless researchers agree on some basic definition of who should be defined as a victim. This is the first subject of discussion. After a short description of the way victimizations occur, the consequences of the robbery are discussed, and the responses of the mental health and justice systems are presented.

Most victims do not resist and those who do so seem to be reacting to past victimizations or to an excess of violence on the part of the robber. Nearly $90 \%$ of victims suffer some kind of emotional trauma and far from being helped in this regard, this trauma is often aggravated by the criminal justice system's response. It seems to affect the victim much more than the financial, physical and social consequences of the crime, which had little effect on their attitudes and needs.

The main problem with armed robbery is that it creates and perpetuates a climate of suspicion, fear and anger very damaging to social relationships. These negative effects can be reduced, however, and the study points out some of the means by which this can be accomplished.

\section{INTRODUCTION}

Le vol à main armée a longtemps été considéré comme un crime contre la propriété. Sa gravité dépendait de l'importance des

* Micheline Baril, professeure, École de criminologic, Université de Montréal.

** Anne Morissette, assistante de recherche, Centre international de criminologie comparée, Université de Montréal. 
sommes volées. Cette mentalité persiste aujourd'hui dans l'opinion publique comme dans les pratiques policières et judiciaires.

Mais les personnes qui ont été les cibles de vols à main armée émettent une opinion très différente : le hold-up, disent-elles, est un crime violent, une atteinte sérieuse à l'intégrité physique. émotionnelle et sociale des individus. Selon les victimes, la perte financière - étalon de mesure utilisé par le système judiciaire actuel - illustrerait bien mal l'impact réel de la victimisation.

Depuis 1976, nous essayons de comprendre la signification du vol à main armée pour ceux qui le subissent directement ou de façon secondaire. Une première série d'études avait pour objectifs principaux de découvrir la perception du crime et de la justice qu'ont les commerçants victimes et de voir de quelle façon ils sont traités par le système de justice (Giroux et Huot, 1977; Grenier et Manseau, 1977; Baril, 1978). Ces travaux ont été réalisés dans le cadre d'une vaste enquête sur l'opinion publique relative aux questions criminelles. En 1980, le Groupe de travail sur les vols à main armée reprit l'enquête qualitative auprès des victimes.

Les études victimologiques entreprises à l'École de criminologie et au Centre international de criminologie comparée de l'Université de Montréal depuis 1980 ont permis d'élargir les perspectives de recherche à d'autres types de victimisation et à d'autres thèmes, notamment les conséquences du crime et les moyens de le prévenir, le rôle des victimes dans le système de justice criminelle, les façons de réparer les préjudices (Baril et Laflamme-Cusson, 1983; Baril et al., 1983; Baril, 1984; Morissette et Baril, 1985).

Dès lors, on connaissait bien les péripéties du vol à main armée, la gamme des émotions vécues par les victimes tout comme la nature des séquelles subies. On savait aussi que le contact avec l'appareil judiciaire est une expérience rare mais éprouvante pour les témoins/victimes. Mais comment diminuer la fréquence des hold-up? Et lorsque l'agression armée se produit malgré tout, peut-on en amortir l'impact? Pour répondre à ces questions, on a utilisé l'approche du sondage, lequel fut réalisé dans le cadre de l'étude globale du phénomène du vol à main armée décrite dans cette publication.

L'analyse des résultats des $\mathbf{4 4 0}$ questionnaires complétés dans des commerces victimes (340) et des commerces non victimes 
(100) de trois districts policiers de Montréal a donné lieu à deux rapports (Morissette, 1984; Poirier, en cours).

Nos propos, dans cet article, seront basés principalement sur les réponses des 182 personnes présentes lors du vol à main armée dans les commerces sélectionnés pour le sondage. Ils seront complétés et illuminés par les résultats des études antérieures et concomitantes.

\section{LES VICTIMES}

D'après le sondage canadien sur la victimisation, 21600 personnes auraient été victimes de vols à main armée en 1981 dans sept grandes villes canadiennes mais à peine plus de la moitié de ces agressions auraient été signalées à la police. Le pourcentage de dénonciations augmente considérablement toutefois lorsque l'arme utilisée est une arme à feu $(83 \%)$.

Les statistiques sur les victimes de vol à main armée sont très incomplètes et souvent obscures. Lorsqu'un particulier est attaqué sur la rue, il n'y a aucune ambiguilté. Quand c'est une entreprise qui est la cible du vol, identifie-t-on comme victime l'entreprise ou les individus qui y travaillent? Et dans ce dernier cas, qui est victime : tous les employés présents lors du hold-up ou seulement ceux qui ont dû faire face au voleur armé? Par exemple, cinq des sept caissières d'un magasin d'alimentation ont dû remettre, sous la menace, l'argent dont elles avaient la garde. Les voleurs ont été obligés de fuir avant de dévaliser les deux autres caisses. Combien a-t-on de victimes? Une, cinq, sept ou huit - en comptant le propriétaire? En effet, le propriétaire absent au moment du crime et donc non confronté à la violence doit-il être compté au nombre des victimes du fait qu'il subit la perte économique? Les études que nous avons consultées ne clarifiant pas ces points, il est impossible de dresser un portrait des victimes à partir d'une revue de la littérature.

Dans le sondage réalisé auprès des petits commerçants de Montréal (Morissette, Baril, Poirier, 1985), le problème a été abordé ainsi : l'unité d'échantillonnage était le commerce victime (il semble que près de la moitié des commerces répertoriés aient subi un hold-up, entre le $1^{\text {er }}$ février 1982 et le $1^{\text {er }}$ mars 1984); à l'intérieur de ces commerces, un répondant présent lors du vol le plus récent était choisi de façon aléatoire pour répondre au 
questionnaire à titre de victime. Si cette façon de procéder ne permet pas de comptabiliser le nombre de victimes, elle peut néanmoins permettre une description acceptable des personnes confrontées directement au vol à main armée.

Qui sont ces personnes? Des femmes dans un peu plus de la moitié des cas et surtout des femmes salariées; les hommes victimes sont beaucoup plus souvent propriétaires ou gérants. Dans les institutions financières et dans les entreprises commerciales, ce sont généralement les femmes qui manipulent l'argent et les hommes qui possèdent ou gèrent les fonds. Ainsi, selon la façon de définir la victime, un sexe ou l'autre prédomine dans les statistiques de victimisation. Dans plus de $40 \%$ des cas, les victimes avaient déjà subi un hold-up au travail, antérieur à celui qui faisait l'objet de l'étude mais très peu d'entre elles avaient fait l'objet de violence à l'extérieur du contexte d'emploi.

Assez naturellement, les commerces les plus vulnérables sont ceux qui sont les plus accessibles au public (heures d'ouverture, taux de fréquentation, facilité d'abord) et semble-t-il, ceux qui emploient le moins de travailleurs. Près des trois quarts des commerces victimisés durant les deux années sur lesquelles portaient l'étude n'en étaient pas à leur première expérience de hold-up. On se rappellera d'ailleurs que les 40 commerçants victimes interviewés en 1977 avaient subi plus de 250 vols à main armée. Certains ne les comptaient plus. Un propriétaire de dépanneur détenait la palme avec 41 hold-up en un peu plus de sept ans. Au cours de diverses études, nous avons rencontré des préposé (e) $\mathrm{s}$ aux caisses si souvent agressés qu'ils ont perdu la trame de leur histoire de victimisation.

En somme, le hold-up est un risque du métier beaucoup plus qu'une résultante de la personnalité ou des caractéristiques des victimes. Les femmes en sont probablement plus souvent les victimes directes, dans les entreprises commerciales, parce qu'elles manipulent la monnaie. La fréquence de la victimisation est liée aux caractéristiques de l'entreprise et à l'obstination des propriétaires et employés à se maintenir en affaires.

\section{LES SCÉNARIOS}

Les vols à main armée se passent très rapidement. Malgré la grande diversité des scénarios qui se déroulent dans un très court 
laps de temps, on peut relever quelques constantes. Les voleurs dans les commerces sont jeunes, ils travaillent seuls ou en duo, ils se donnent peu de mal pour déguiser leur identité, ils ont surtout recours aux armes à feu mais ils agissent de façon discrète. C'est au cinéna qu'on peut observer les grands déploiements de violence. Dans la vie quotidienne, le hold-up est un événement qui passe inaperçu des témoins le moindrement distraits; ce caractère banal et apparemment anodin ressort bien dans les vidéocassettes collectionnées par le service de police ${ }^{1}$. Près de $60 \%$ des répondants du sondage réalisé à Montréal n'ont pas perçu l'approche du ou des voleurs comme particulièrement violente. D'ailleurs, dans plus de $95 \%$ des situations, aucun élément spectaculaire n'a pris place; il n'y eût ni coups de feu, ni cris, ni séquestration, ni aucune autre forme d'intimidation voyante.

Terne pour le spectateur, le hold-up n'en suscite pas moins quelques instants de frayeur chez les victimes directes. D'abord, aussi averti qu'on soit, on ne s'y attend jamais, pas à ce moment-là. Ensuite, la présence d'une arme domine la situation même lorsque cette arme est invisible ou simulée. En 1977, nous constations avec étonnement que plusieurs victimes s'étaient posé la question de l'authenticité du revolver : est-ce un jouet ou une arme réelle? Depuis, de nombreuses autres personnes nous ont communiqué ce même questionnement. Il semble que la situation soit perçue comme trop menaçante pour être réelle, et trop banale pour être menaçante. Tout récemment, une caissière exprimait cette ambivalence ainsi :

Tout ce que j'ai vu, c'est le trou noir du revolver et j'ai figé. Mais, ça se passe comme un flash, tu te dis que c'est impossible, tu dois être au cinéma, ailleurs, ça ne peut pas être une vraie arme, c'est une farce, ça doit être un jouet. N'empêche que j'ai figé. Je voyais seulement le trou noir 2 .

Pour l'agresseur aussi, la situation est hautement volatile. Ces quelques minutes concentrent des risques de tous genres, et

1. Les magasins plus vulnérables, comme beaucoup d'institutions financières, sont souvent équipés de caméras qui enregistrent notamment les vols à main armée.

2. Tiré d'une entrevue, réalisée par une étudiante stagiaire en 1985. On peut aussi consulter Grenier et Manseau (1977), Baril (1978), Baril (1984). 
peut-être également un sentiment d'exaltation. Quoiqu'il en soit, les victimes observent un état général de nervosité chez les voleurs et cette constatation ne les rassure guère (Grenier et Manseau, 1977).

Ayant posé un premier geste, planifié dans une certaine mesure, l'agresseur contrôle la situation au départ. Cependant, il ne peut prévoir les réactions, spontanées ou calculées, de ses victimes, ni les événements inopinés qui risquent de se produire durant les quelques minutes de l'agression (arrivée de témoins ou même de la police, sonnerie du téléphone, problèmes techniques d'un tiroir-caisse qui refuse d'ouvrir, ...). . De tels événements peuvent prendre l'agresseur au dépourvu et renverser la situation de pouvoir.

En effet, le vol à main armée dans un établissement commercial est une expérience très spéciale pour toutes les parties impliquées; expérience très vitale également puisque chacun y risque sa vie ou son intégrité en quelques instants dramatiques. La rapidité des échanges empêche toute stabilisation des rôles selon des modèles déjà apprivoisés; les réactions sont instinctives, improvisées, primitives.

TABLEAU 1

La première réaction de la victime

\begin{tabular}{lrc}
\hline Réaction & $\mathrm{N}$ & $\%$ \\
\hline Obéissance immédiate & 108 & 59,3 \\
Hésitation, paralysie temporaire, incrédulité & 45 & 24,7 \\
Refus, résistance & 12 & 6,6 \\
Éclatement émotif & 17 & 9,3 \\
Total & 182 & 100,00 \\
\hline
\end{tabular}

L'élément de surprise appelle des réponses quasi instinctives. De plus, les commerçants disent, lors d'entrevues en profondeur. que la vie a beaucoup plus d'importance que l'argent. Il importerait dès lors de ne pas prendre de risques. Mais comment, alors expliquer le comportement des victimes qui ne se soumettent pas au départ? Et, surtout, comment interpréter le fait que durant un 
vol qui se déroule très rapidement, bon nombre de victimes optent finalement pour la résistance?

Le sondage de 1984 , à Montréal, révèle que $30 \%$ des victimes disposées à la soumission au début du hold-up ont changé d'avis durant le déroulement du vol et ont manifesté ensuite une résistance. Par ailleurs, toutes les victimes qui ont, dès le départ, refusé de se soumettre, ont maintenu leur décision.

TABLEAU 2

La résistance de la victime selon sa première réaction

\begin{tabular}{lcccccccccc}
\hline & \multicolumn{1}{c}{ Obéissance } & \multicolumn{1}{c}{ Hésitation } & \multicolumn{1}{c}{ Refus } & \multicolumn{1}{c}{ Éclatement } & \multicolumn{2}{c}{ Total } \\
Résistance & $\mathrm{N}$ & $\%$ & $\mathrm{~N}$ & $\%$ & $\mathrm{~N}$ & $\%$ & $\mathrm{~N}$ & $\%$ & $\mathrm{~N}$ & $\%$ \\
\hline Oui & 12 & 11,1 & 11 & 24,4 & 12 & 100 & 10 & 58,8 & 45 & 24,7 \\
Non & 96 & 88,9 & 34 & 75,6 & & & 7 & 41,2 & 137 & 75,3 \\
Total & 108 & 100 & 45 & 100 & 12 & 100 & 17 & 100 & 182 & 100 \\
\hline
\end{tabular}

Malheureusement, nos données ne parlent pas au-delà des questions qui ont été explicitement posées. Retenons que le quart des victimes ont opposé une résistance à l'agression durant son déroulement alors que seulement $7 \%$ avaient spontanément réagi ainsi dès l'amorce du hold-up. Et pourquoi les victimes changeraient-elles ainsi d'idée? Certaines ont eu la chance de se ressaisir. Mais plusieurs, semble-t-il, ont été indignées par un excès de violence de la part du voleur. On pourrait donc recommander aux agresseurs d'user de sobriété dans leurs menaces. Mais que conseillera-t-on aux victimes?

Les femmes résistent un peu plus que les hommes mais ont tendance à adopter des formes passives de résistance. Autant les employés que les patrons refusent de se soumettre à l'ordre de livrer les recettes, autre indice de l'importance secondaire que prennent les pertes matérielles. Ce sont surtout les commerçants qui ont été victimisés antérieurement qui opposent un mode ou un autre de résistance. Il semble que la tolérance diminue avec le temps. C'est d'ailleurs ce que les victimes nous ont dit en entrevue. Certains commerçants croyaient à l'effet dissuasif de la résistance. Selon eux, les voleurs échangeraient des renseignements quant à la malléabilité de leurs proies et s'attaqueraient ainsi, démesurément, à celles qui obéissent aux ordres. 
Si on se laisse voler, il n'y aura plus de fin.

Dans les entretiens en profondeur, les commerçants expriment surtout leur désir de protéger leur vie et leur intégrité physique avant de conserver leur argent. Ils auraient raison en ce que la résistance, bien qu'elle diminue les probabilités de pertes financières, augmente les risques de blessures. Les résultats de l'étude de 1984 vont dans ce sens quoique l'échantillon soit trop restreint pour permettre des conclusions fiables. Block (1981) est cependant arrivé aux mêmes résultats à partir de l'analyse d'un grand nombre de cas.

Il faut bien se rappeler les composantes essentielles du hold-up : présence d'une arme, effet de surprise, nervosité de l'agresseur, rapidité des séquences, événements imprévus. Dès lors, il n'est pas surprenant que les parties adoptent des comportements parfois bizarres, parfois en flagrante contradiction avec leurs projets les plus fermes. L'analyse rétrospective et froide des phénomènes d'autodéfense néglige généralement la réaction émotive, incontrôlable, irrationnelle, de l'individu qui, après avoir longtemps contemplé et ruminé diverses réponses à l'agression, se retrouve dans une situation menaçante. On aura beau interpréter après coup mais comment comprendre?

\section{LES CONSÉQUENCES}

La majorité des études en victimologie constatent que l'expérience de victimisation marque ceux qui la subissent. Baril (1980) a déjà observé des similitudes entre les réactions des victimes d'agression sexuelle et les comportements de victimes d'autres types de crimes. Il semble bien que la perte de pouvoir, ou encore l'impuissance ressentie par l'agressé au moment de l'attaque engendre presque toujours un certain déséquilibre, dont les manifestations toutefois peuvent varier. Les symptômes d'un traumatisme imputable à la victimisation peuvent être physiques ou psychologiques (maux de tête, nausées, nervosité, dépression, agressivité, etc.). Ces perturbations risquent de se répercuter sur la vie familiale et sociale des agressés. On constate souvent que l'entourage des victimes est également affecté.

Il n'est pas suffisant de connaître la nature des séquelles, il faut aussi en avoir la fréquence et le mode de répartition. Sur ces derniers points, les connaissances sont plutôt pauvres et une des 
visées de l'étude était de faire progresser la question en vue éventuellement de diminuer ou de réparer les préjudices.

À partir des quatre types de séquelles des victimisations, il s'agissait de voir dans quelle mesure les victimes de vol à main armée étaient touchées, pourquoi et comment.

\section{PERTES FINANCIĖRES}

C'est là l'aspect le plus visible du hold-up. Pourtant, ce n'est pas toujours ce qui préoccupe le plus les victimes.

Lorsque le vol prend une ampleur plus menaçante, soit par sa durée, soit par l'agressivité déployée, les considérations financières s'estompent (Manseau, 1980, p. 60).

Les résultats de l'enquête démontrent que le hold-up a réussi, c'est-à-dire que le bandit s'est enfui avec une somme d'argent quelconque dans 162 cas, ou $90 \%$ des vols. Il est remarquable de constater que $50 \%$ des délits rapportent moins de $200 \$$ aux voleurs, et que parmi ceux-ci, $28 \%$ ne dépassent pas le cap de $100 \$$. Il est clair que le vol à main armée dans les commerces ne permet pas aux bandits de faire fortune, ce qui fait dire à plusieurs victimes : «tout ça pour si peu...»

En plus des pertes d'argent liquide, certaines victimes ont été délestées de leurs effets personnels $(9,3 \%)$. Parfois (19\%) les bandits pouvaient s'enfuir avec de la marchandise (bijoux. drogues, etc.). D'autres ont mentionné des dommages matériels plus ou moins importants (téléphone arraché, caisse brisée, etc.). Cependant, pour une majorité de victimes, le montant des pertes directes n'était pas très élevé. De plus, la plupart des victimes étaient assurées. Par contre, les commerçants n'ont pas tous déclaré leurs pertes à leur compagnie d'assurances, par crainte de voir leur prime augmenter. L'augmentation du coût des dépenses policières, l'accroissement de l'investissement en matière de prévention, la hausse des primes d'assurances ou encore la diminution des heures d'ouverture d'un commerce font partie des pertes indirectes imputables au vol à main armée. S'il est difficile d'y accoler un chiffre exact, on ne doit pas les négliger pour autant.

Le montant des pertes financières, directes ou non, attribuables au vol à main armée est parfois un piètre indicateur de l'impact véritable de ce crime. En effet, lorsqu'on évalue le coût du hold-up, on doit le confronter à la capacité d'absorption réelle de 
chacun, à court et à long terme. Une perte de $200 \$$ par exemple, n'implique pas la même chose pour toutes les victimes. Ce facteur, tout aussi relatif qu'il soit, enrichirait l'analyse des conséquences du hold-up.

\section{PERTURBATIONS PHYSIQUES}

Après un vol à main armée, il est possible que les victimes soient atteintes de malaises physiologiques, et ce en sus des blessures infligées par les agresseurs $(4,9 \%)$. Nos résultats démontrent que $61 \%$ des répondants souffrent de perturbations comme la nervosité chronique et les troubles de sommeil, tandis que $39 \%$ des victimes ne mentionnent aucune indisposition particulière. Ces chiffres sont assez étonnants puisque les résultats antérieurs ne les laissaient pas présager. En effet, la plupart du temps, le vol s'était déroulé rapidement sans trop de complications, pour le bandit bien entendu, et sans que la victime ne soit malmenée dans une majorité de cas. D'ailleurs cette tendance est encore plus significative lorsqu'on analyse l'occurrence des perturbations psychologiques. En effet, les victimes sont bouleversées par le hold-up, et c'est généralement l'impact le moins visible de ce crime.

\section{PERTURBATIONS PSYCHOLOGIQUES}

Pendant le déroulement du vol à main armée, une fois le moment de surprise passé, le sentiment prédominant est la peur. La plupart des victimes craignent pour leur intégrité physique. L'arme braquée contre elles, cette menace explicite, suscite une angoisse, un stress, quand ce n'est la panique. Le contrôle de la situation est dans les mains d'un inconnu armé.

Il n'est pas surprenant que les victimes éprouvent un choc nerveux durant l'agression ou immédiatement après. C'est le contraire qui étonne : quelques travailleurs dans les commerces réagissent avec un remarquable sang-froid et semblent surmonter assez facilement les effets d'une expérience qui n'en demeure pas moins traumatisante. De leurs témoignages, on pourrait croire qu'avec le temps on s'habitue. C'est vrai pour certains.

Puisque la plupart des victimes vivent très intensément l'agression, il est naturel qu'elles en soient marquées, changées, pour le meilleur ou pour le pire. Chez les personnes interrogées 
lors du sondage, $89 \%$ ont fait état de perturbations psychologiques à moyen ou à long terme. Leurs réponses sont inquiétantes. II semble que le vol à main armée, loin de susciter une cohésion sociale à la manière de Durkheim, favorise un effritement des rapports sociaux. En effet, $78 \%$ des victimes de hold-up deviennent méfiantes à l'égard d'autrui et $72 \%$ redoutent davantage le vol violent (tableau 3).

TABLEAU 3

La présence de perturbations psychologiques

\begin{tabular}{lrrrrrr}
\hline & \multicolumn{3}{c}{ Présence } & & \\
Nature des perturbations & \multicolumn{2}{c}{ Oui } & \multicolumn{2}{c}{ Non } & \multicolumn{2}{c}{ Total } \\
& $\mathrm{N}$ & $\%$ & $\mathrm{~N}$ & $\%$ & $\mathrm{~N}$ & $\%$ \\
\hline Peur des hold-up & 131 & 72,0 & 51 & 28,0 & 182 & 100 \\
Méfiance accrue envers autrui & 142 & 78,0 & 40 & 22,0 & 182 & 100 \\
Agressivité/colère & 46 & 25,3 & 136 & 74,7 & 182 & 100 \\
Fréquents changements d'humeur & 25 & 13,7 & 157 & 86,3 & 182 & 100 \\
Dépression & 18 & 9,9 & 164 & 90,1 & 182 & 100 \\
Peur généralisée & 24 & 13,2 & 158 & 86,8 & 182 & 100 \\
Autres & 5 & 2,7 & 177 & 97,3 & 182 & 100 \\
Aucune perturbation & 20 & 11,0 & 162 & 89,0 & 182 & 100 \\
\hline
\end{tabular}

Il semble bien, en fait, que presque toutes les victimisations violentes déclenchent des réactions émotives de durée et d'intensité variables. Par exemple, au Centre d'aide aux victimes d'actes criminels du quartier Hochelaga-Maisonneuve de Montréal, on a trouvé que $84 \%$ des victimes d'agressions souffraient de perturbations psychologiques (Morissette et Baril, 1985). Cette étude incluait, outre les victimes de vols qualifiés, des personnes ayant subi des voies de fait, des agressions sexuelles, des tentatives de meurtre, des délits contre la propriété. La violence, ou le degré perçu de violence serait donc la principale raison de l'émergence de traumatismes.

Il n'y aurait peu ou pas de relations entre la perte financière et la perturbation émotive. Par exemple, employés et propriétaires éprouvent des difficultés émotionnelles, dans une même proportion (Morissette, 1984). 
Les hommes vivent l'agression aussi péniblement que les femmes, ou presque (Morissette, 1984). Soulignons ici que la plupart des hommes, étant majoritairement propriétaires des commerces, subissent une double victimisation.

À partir des propos des victimes recueillies depuis plusieurs années (Baril, 1984), nous nous attendions à ce que celles qui résistent à l'agression voient leur santé mentale moins détériorée que celles qui se soumettent. Les premières, pouvant conserver une bonne mesure d'estime personnelle, pouvant même se féliciter de leur bravoure, seraient en mesure de se ressaisir beaucoup plus rapidement que les secondes. Les résultats de l'étude de 1984 sur le vol à main armée n'appuient pas cette hypothèse. Peut-être parce que notre échantillon comporte un faible nombre de victimes qui ont opposé une résistance $(24 \%)$, alors que le pourcentage de victimes traumatisées est très élevé $(89 \%)$. Peut-être aussi qu'il s'agit d'une particularité du vol à main armée auprès de commerçants, d'autres types de victimes ressentant des émotions différentes. Il ne faut pas exclure non plus la possibilité que la perte d'estime de soi ou le sentiment vécu d'impuissance aient une influence limitée sur l'évolution des séquelles psychologiques. Voilà une autre question pour les recherches ultérieures.

Puisque $90 \%$ à peu près des victimes éprouvent des difficultés d'ordre psychologique, il est très difficile d'identifier des facteurs discriminants à moins de mesurer de façon précise l'intensité et la durée des difficultés décrites. L'instrument de cueillette des données ne permet pas d'évaluer la gravité des séquelles. Quant à leur durée, on constate chez la moitié des victimes des troubles qui persistent depuis au moins sept mois. Ce résultat est d'autant plus alarmant que le vol investigué était le plus récent durant les deux dernières années.

Si on conjugue les éléments "durée» et «intensité» dans chacune des deux études quantitatives réalisées dans les mêmes secteurs géographiques de Montréal (Morissette, 1984 et Morissette et Baril, 1985), deux facteurs de traumatismes se dégagent. L'un est la victimisation antérieure qui développerait une fragilité, ou une immunité réduite. L'autre est la présence de difficultés personnelles ou sociales au moment de la victimisation (faiblesse des liens sociaux, deuil, problèmes économiques, ...). Il faudra aller beaucoup plus loin sur ces pistes de recherche. 
Il faut retenir que l'impact le plus important du vol à main armée se produit au plan de la santé mentale des individus agressés. On connaît peu les raisons pour lesquelles des victimes de crimes semblables réagissent de façons différentes. Mais, de plus en plus, on devient conscient de l'effet de contagion de la violence subie.

\section{IMPACT SUR LA VIE SOCIALE}

L'impact social du vol à main armée n'est pas facile à évaluer. Si les conséquences psychologiques du hold-up sont assez bien circonscrites, leurs manifestations concrètes sur la vie sociale des individus demeurent assez vagues. On sait que les victimes les plus traumatisées n'ont probablement pas répondu au questionnaire. Elles ont tout simplement refusé, changé d'emploi ou même déménagé. Malgré cela, les résultats nous permettent de croire que le hold-up a une influence importante à la fois sur la qualité de vie individuelle et sur le bien-être collectif.

Par exemple, $20 \%$ des répondants du sondage de 1984 ont révélé avoir vécu des difficultés personnelles après le crime. La vie familiale et sociale risque donc d'être bouleversée. Les résultats de l'étude AVI (Morissette, Baril, 1985) sont encore plus probants. En effet, $80 \%$ des clients du Centre d'aide AVI ont vécu des problèmes sociaux, variant de la peur de sortir de chez soi, à la rupture des liens familiaux ou sociaux, au déménagement. Le quotidien de ces individus, leur style de vie, a dû être modifié suite à la victimisation. L'écart entre ces résultats peut s'expliquer d'une part par des erreurs de mesures dans l'une ou l'autre des études, ou d'autre part par des différences fondamentales entre les types de victimes. En effet, il est possible que les victimes de vol à main armée ressentent moins concrètement les manifestations directes de l'impact social de ce crime que les autres victimes de violence grave, clientes d'AVI. Dans le premier cas, le malaise est beaucoup plus diffus, et adopte moins fréquemment une forme aussi précise que la peur de sortir de chez soi. Il est permis de croire que pour les victimes de vol à main armée une perturbation psychosomatique ou l'accroissement de la méfiance envers autrui n'a pas un impact immédiat et apparent sur la qualité de vie personnelle et sur le bien-être collectif. Son influence presque imperceptible à court terme se traduirait plutôt par une lente altération des attitudes, comme, par exemple, la perte de confiance envers le système de justice. 


\section{LES INTERVENTIONS}

Selon le sondage canadien sur la victimisation, $83 \%$ des vols commis au moyen d'une arme à feu seraient signalés à la police. À Montréal, lorsque ce type de vol est commis contre des petits commerces, le pourcentage de dénonciation est encore plus élevé : $98 \%$, dans le sondage de 1984 (Morissette, 1984). Il faut préciser que ce sondage a été réalisé dans des quartiers où la police avait établi des relations privilégiées avec les commerçants.

Il nous semble que les relations soient au beau fixe entre la police et les commerçants victimes, depuis les dix dernières années. Les résultats de notre dernier sondage ajoutent peu aux études antérieures : en général, les victimes aiment bien la police même si elles expriment beaucoup de réserves quant à son efficacité.

Le taux de solution des vols à main armée contre les petits commerces étant très faible (moins de $25 \%$ ), peu de causes sont portées à l'attention du tribunal. Parmi celles-ci, bien souvent la victime n'est pas impliquée parce que l'accusé plaide coupable à l'accusation initiale ou à une accusation moindre. Ainsi, seulement $7 \%$ des 182 victimes directes interviewées ont eu un contact avec le système judiciaire et ont pu exprimer leurs commentaires.

\&La justice, quelle justice?» se demandent les victimes qui ont dû témoigner. Cette interrogation fut enregistrée en 1977. Elle n'a cessé d'être répétée depuis sous de multiples variations. Les victimes se sentent ignorées par l'appareil pénal, dépossédées, abusées, négligées. La voix des victimes du sondage montréalais de 1984 est trop faible pour illustrer ces insatisfactions qui paraissent toutefois très clairement dans les entrevues réalisées antérieurement.

Au plan psychosocial, l'intervention a connu un essor récent, mais bien modeste. Il existe maintenant des services d'aide aux individus victimes. Services éphémères peut-être, services souséquipés certainement. Néanmoins, on a constaté, à Montréal, que $75 \%$ des victimes de violence, - la proportion est encore plus élevée dans les cas de vols qualifiés - pouvaient se tirer d'affaires seules ou avec une intervention minimale de soutien émotif et d'information; cette assistance est nommée «préventive». Elle était offerte dans deux des trois districts policiers où s'est déroulée l'enquête mais la plupart des victimes interviewées avaient subi leur hold-up avant que les centres d'aide ne soient mis sur pied. Environ $15 \%$ des victimes de vol qualifié auraient besoin d'une 
intervention plus intensive mais peu d'entre elles auraient accès à des spécialistes en santé mentale aptes à les assister.

\section{CONCLUSION}

Du point de vue de ceux qui le subissent, le vol à main armée est un crime de violence contre la personne et non un délit économique.

Il affecte les victimes immédiates surtout dans leur intégrité émotive mais aussi dans leur mode de vie et dans leurs relations de travail. Parfois, il détruit l'intégrité physique. Pour un petit nombre d'individus, il occasionne des difficultés financières majeures. Les faibles gains, pour les voleurs, sont tout à fait disproportionnés avec les pertes, pour les victimes. L'intervention du système de justice risque d'accroître ces pertes. Par ailleurs, le réseau psychosocial fait très peu pour aider les victimes à guérir leurs blessures.

Un des objectifs de la recherche était de prévenir ou de diminuer les traumatismes chez les victimes. Qu'avons-nous appris? Que pouvons-nous dire aux victimes et aux intervenants?

Mieux vaut obéir aux voleurs, semble-t-il. Si la soumission augmente les risques de pertes économiques (et on peut toujours réduire les liquidités en caisse), elle diminue le danger de violence grave sans affecter, pour autant, le sentiment d'estime de soi. Ceci, la majorité des commerçants en ont déjà l'intuition. Mais l'écart est grand entre le savoir et le faire car le vol à main armée demeure un événement imprévisible, qui se déroule très rapidement et soulève beaucoup de crainte et de colère.

Environ le quart des commerçants victimes ont résisté à un moment ou l'autre du vol. Si cette proportion augmentait, si les victimes devenaient plus agressives, pourrait-on espérer une diminution du vol à main armée, les voleurs prenant conscience des risques encourus? C'est surtout répondre à la violence par une violence accrue. Dans les faits, les victimes sont rarement disposées à apporter une résistance agressive.

Enfin, il y aura toujours des vols avec violence; la grande majorité de ceux qui les subissent en seront traumatisés. On s'entend généralement pour dire qu'il faut guérir ce qu'on n'a pu prévenir. Ici, la société a un choix beaucoup plus étendu de 
mesures efficaces, de l'augmentation du taux des crimes, à un service de justice humanisé, à des services d'aide psychosociale. Puisque la méfiance à l'égard d'autrui est la séquelle la plus souvent observée suite au hold-up, c'est la qualité de la vie sociale qui est menacée par la violence. Une considération accrue à l'égard des victimes saurait-elle restaurer un climat plus sain?

\section{RÉFERENCES}

BARD, M., SANGREY, D. (1980), The Crime Victim's Book, New York, Basic Book.

BARIL, M. (1978), «New direction in victim research : victim's perception of crime and criminal justice system», Unpublished M.Ph.L. Thesis, Cambridge, University of Cambridge (publiée en partie dans Block, R. (édit.) (1984), Victimization and Fear of Crime: World Perspectives. Washington, D.C., U.S. Department of Justice) .

BARIL, M. (1980a), Rape and Other Acts of Violence, Paper read at the meeting of the American Society of Criminology in San Francisco, California, novembre, 4-10th, 1980.

BARIL, M. (1980b), «Ils n'ont plus la liberté : réactions à la victimisation et ses conséquences», Criminologie, 13, 1, p. 94-103.

BARIL, M., S. DURAND. M.M. COUSINEAU, et S. GRAVEL (1983a), «Mais nous, les témoins...», Étude exploratoire des besoins des victimes et témoins, Montréal, École de criminologie, Université de Montréal.

BARIL, M., S. LAFLAMME-CUSSON (1983b), I'Indemnisation des victimes d'actes climinel : une évaluation du service québécois. Montréal. École de criminologie et Ottawa, ministère de la Justice, «Victime d'actes criminels».

BARIL. M. (1984), «L'envers du crime», les Cahiers de recherches criminologie, no 2, Centre international de criminologie comparée, Université de Montréal.

BLOCK, R. (1981), «Victim Offender Dynamic in Violent Crime», Journal of Law and Criminology, vol. 72, $\mathrm{n}^{\circ}$ 2, p. 743-765.

DRAPKIN, I., E. VIANO (édit.) (1974-1975), Victimology : A New Focus, 5 vol., Lexington, Mass., Lexington Books.

FATTAH, E. (1984), «Victims Response to Confrontational Victimization. A Neglecter Aspect of Victim Research», Crime and Delinquency, vol. $30, n^{\circ} 1$, p. $75-89$.

FRIEDMAN, K., R. BISCHOFF, R. DAVIS, A. PERSON (1982), Victims and Helpers: Reaction to Crime, New York, Victim Services Agency.

GALAWAY, B., J. HUDSON (1981), Perspectives on Crimes Victims, St-Louis, Missouri, the C.V. Mosby company. 
GIROUX, J., L. HUOT (1977), «Le citoyen victime de vol qualifié : sa place dans le processus judiciaire», G.R.A.C., rapport no 5 , Montréal, Centre international de criminologie comparée, Université de Montréal.

GRENIER, H., D. LEMAIRE, H. MANSEAU (1977), «Les petits commerçants victimes de vol à main armée : En quête de justice», G.R.A.C., rapport no 5 , Montréal, Centre international de criminologie comparée, Université de Montréal.

GROUPE DE TRAVAIL SUR LE VOL A MAIN ARMÉE (1980), (Rapport Laplante), le Vol à main armée au Québec, Québec, Bibliothèque nationale.

GROUPE D'ÉTUDE FÉDÉRAL-PROVINCIAL CANADIEN SUR LA IUSTICE POUR LES VICTIMES D'ACTES CRIMINELS (1983), Rapport final, Ottawa, ministère des Approvisionnement et services.

HINDELAND, M.I., M.R. GOTTEFREDSON, I. GAROFALO, (1978), Victims of Personal Crime :An Empirical Foundation for a Theory of Personal Victimization, Cambridge, Mass,, Ballinger Publishing Company.

LEGER, G. (1982) , «La victime du crime», Impact, n 1, Ottawa, ministère du Solliciteur général.

MAGUIRE, M. (1980), «The Impact of Burglary upon Victims», The British Journal of Criminology, vol. 20, no 3, p. 261-275.

MANSEAU, H. (1980), «Les vols à main armée tels que vus par les victimes», Groupe de travail sur le vol à main armée, Annexe no 3 , Université de Montréal, Centre international de criminologie comparée.

MIDDENDORF, W. (1977), «Bank Robbers and Their Victims», Annales internationales de criminologie, vol. 16 , (1 et 2), p. 179-232.

MINISTĖRE DU SOLLICITEUR GÉNÉRAL (1984-1985), Bulletin sur le sondage canadien de victimisation en milieu urbain. Ottawa, ministère du Solliciteur général.

MORISSETTE, A. (1984), «Subir un vol à main armée : réactions et conséquences», Mémoire de maîtrise, École de criminolokie, Université de Montréal.

MORISSETTE, A. et M. BARIL (1985), «Le centre d'aide aux victimes AVI : Rapport d'activités et de recherches; année 1984», Ottawa, ministère du Solliciteur général.

POIRIER, D. (en cours), la Prévention et le vol à main armée, Mémoire de maîtrise, Êcole de criminologie, Universitê de Montréal.

SALASIN, S.E. (édit.) (1981), Evaluating Victim Services, Beverly Hills, Calif., Sage Publication.

SCHNEIDER, H.J. (édit.) (1982), The Victim in International Perspective, Berlin, New York, De Gruyter.

SPATES, R. (1982), «The Mental Health Needs of Victims : A Review of the Literature and Recommandation for Research», Prepared for the National Institute of Mental Health.

SYMONDS, M. (1980), «The Second Injury to Victims», Evaluation and Change : Special Issues, Minneapolis, p. 36-42.

WALLER, I. (1981), «Les victimes d'actes criminels : Besoins et services. Canada/États-Unis», Déviance et société, vol. 5, no 3, pp. 263-276. 